\title{
Impact of Multi-sensory On-Bicycle Rider Assistance Devices on Rider Concentration and Safety
}

\author{
Chao-Yang Yang ${ }^{1}$, Yu-Ting $\mathrm{Wu}^{2}$, and Cheng-Tse $\mathrm{Wu}^{3}$ \\ ${ }^{1}$ Department of Industrial Design, Tatung University, No.40, Sec. 3 , \\ Zhongshan N. Rd., Taipei City 104, Taiwan \\ dillon.yang@gmail.com \\ ${ }^{2}$ Department of Industrial Design, Chang Gung University, 259, \\ Wen Hua 1st Rd, Kuei Shan, Tao-Yuan, Taiwan \\ hope790406@gmail.com \\ ${ }^{3}$ Department of Industrial Design, Chang Gung University, 259, \\ Wen Hua 1st Rd, Kuei Shan, Tao-Yuan, Taiwan \\ woolmonster@gmail.com
}

\begin{abstract}
This study evaluated the impact of multi-sensory information cues from on-bicycle rider information assistance devices (OBRAD) on hazard perception performance. Experiments tested the impact of distraction from different combinations of visual, auditory and tactile sensory aids on the subject's ability to maintain peddling frequency while conducting eight different tasks. The results indicate that the integrated use of different sensory cues (e.g., text, audible alerts and vibration) can decrease cognitive loading, with each sensory combination, particularly those involving tactile stimulation, having different levels of effect. Tactile sensory aids helped reduce the degree of rider distraction, thus helping maintain a high sensitivity to danger (hit rate mean: 0.34 ). Cycling performance was further improved through combining tactile stimuli with auditory cues for assistance in the secondary task. The implications of these findings and the need to integrate and manage complex OBRAD information systems are discussed.
\end{abstract}

Keywords: cycling performance, multi-sensory, hazard perception, cognitive loading.

\section{Introduction}

Increased popularity of recreational bicycling has driven sales of On-Bicycle Rider Assistance Devices (OBRADs), which offer an increasingly broad range of information, including location and route information (including altitude and slope gradient), and physiological monitoring data including heart rate and cadence.Use of OBRADs can impact the rider's speed control, reaction time, ease of learning and level of psychological stress in emergency situations. Rockwell (1988) recommended a "twosecond rule" for OBRAD use, suggesting that any device which requires drivers to look away from the road for more than two seconds has a significantly negative impact on safety. In consideration of the rider's posture and the design of bicycle 
frames, OBRADs are usually placed on the handlebars to optimize access and visibility to the rider. However, the rider's pedalling action has a constantly shifting impact on his/her line of sight, level, and observational capacity.

The impact of multitasking on driver performance and safety has been widely studied (Salvucci, 2002), but little attention has been focused on the use of OBRADs by cyclists, thus this study begins by reviewing the relevant literature for automotive OBRAD use. Cycling and driving are similar in that they are both multitasking activities primarily concerned with the safe transport of the rider/driver, and both require training to successfully accomplish subtasks including observing road conditions and maintaining constant speed. Lansdown et al. (2004) suggested that driving task demands are variable, and at times the driver may be left with considerable spare capacity which can be used to undertake secondary or dual tasks. Use of OBRADs and similar devices significantly increases the amount of visual information riders must process, thus increasing the potential for distraction and accidents. For example, reading route guidance systems is likely to considerably increase rider's cognitive loading and cause distraction. Given that cyclists today can reach speeds of 40-60 kph, potential harm due to distraction is no less serious for cyclists than for car drivers and entails a risk of serious injury or death. Previous research has shown that cyclists are highly prone to single-driver accidents, often resulting from behavior including excessive speed, lack of attention, breach of traffic regulations or poor co-ordination (Eilert-Petersson \& Schelp, 1997). In addition, "looked, but didn't see" is a puzzling but common type of error, potentially caused by negligence and poor judgment (Stanton \& Salmon, 2009).

Receiving and processing riding information occupies physiological resources, including visual, operational, cognitive and auditory. Previous studies have shown that engagement in non-essential activities degrades driver performance, with contributing factors including attentional allocation (Matanzo \& Rockwell, 1967), driving experience (Summala et al., 1998), age (Brouwer et al., 1991; Korteling, 1994) and mental workload (Hancock \& Verwey, 1997). Dingus (2006) suggested that drivers cannot drive safely while dividing their attention between multiple visual stimuli, and Droll (2011) found that alternating one's gaze between the road and the radio leaves drives vulnerable to collisions. Gopher (1990) suggested that increasing the difficulty of the primary task increases the impact of multi-tasking on overall performance, potentially resulting in fatally delayed response times. However cognitive loading is difficult to measure directly, and is thus typically assessed through the use of subjective ratings or measurements of physiological responses. Studies of drivers' visual attention indicate that night time and rainy driving conditions increase cognitive demand, which thus may reduce the useful field of view (Konstantopoulos et al., 2010). Others have shown that mental workload affects driving performance (Lee et al., 2007; Recarte \& Nunes, 2003) resulting in increased response times (Plainis \& Murray, 2002).

Human reactions to their surroundings can be broken down into three stages: stimuli recognition, reaction choice and reaction step. The process of reaction, from stimulus input through message processing and control operation to reaction output, is a holistic operation and the time required to execute this process is referred to as total reaction time (TRT). From an operational perspective, TRT can be split into reaction time (RT) and movement time (MT), such that TRT $=$ RT+MT $($ Sagberg \& 
Bjørnskau, 2006). Gopher (1990) suggested that adding a secondary task to a difficult primary task can exceed the operator's central processing capacity, thus significantly decreasing primary task performance. Empirical support was provided by Salvucci (2002) who demonstrated that driving involves continual multitasking of a number of sub-processes which show a clear (and significant) task effect, with reaction times increasing from 1.6 seconds to 2.2 seconds when a second task is added.

One's ability to foresee potential traffic accidents is referred to as "hazard perception", and explains the difference between novice and experienced drivers in dealing with distractions without causing collisions (Sagberg \& Bjørnskau, 2006). Some investigations of hazard perception have assessed traffic conditions prior to accidents. These studies used a variety of images (Tränkle et al., 1990), vocal descriptions (Guerin, 1994), video (Renge, 1998) or actual traffic flows (Bragg \& Finn, 1985) to assess hazard perception. Other studies have investigated driver reaction time as a way to assess the likelihood of collision. Some of these studies were based on physical models of traffic situations (CURRIB, 1969) while others used video (Congdon, 1999; McKenna \& Crick, 1997).

Hazard perception studies can be broadly divided into two types: the first primarily focuses on investigating the scene of the potential hazard, while the second is primarily concerned with investigating driver reaction times. The present study is concerned with whether cyclists' hazard perception and reactions undergo significant changes when using OBRADs; thus this study is primarily concerned with the second approach to hazard perception. The present study uses a virtual cycling environment combined with real bicycles to identify the key risk factors for concentration impairment to provide a reference for the formulation of traffic safety standards. In addition to assessing the degree of distraction caused by the use of OBRADs, we also investigate the use of different information reception modes to determine which mode best reduces riders' psychological loading, thus reducing distraction and the risk of collision. This research also measures riders' visual concentration during instances of head movement. Doshi and Trivedi (2008) showed that head movement enables drivers to receive correct information and thus improve the accuracy of their judgments vis-àvis potential hazards.

Warning signals and stimulus signals can be conveyed as visual, auditory or tactile cues through lights, buzzers, vibration or text (Magill \& Anderson, 2007). Auditory stimuli are characterized by short reaction time, judgment direction, distance perception and high conspicuousness. Jones and Furner (1989) noted that, "audition is intimately connected with the arousal and activation systems of the nervous system," indicating that sound can produce faster reaction times than visual stimuli (Welford, 1980). Equally important is that auditory cues can be perceived without the use of either eyes or hands, making them an important means of communication in everyday life and a potentially superior form of alert for OBRADs. Using a driving simulator, Van Erp and Van Veen (2004) found that assistive information (such as alerts) communicated through vibration reduces visual loading. Qian et al. (2011) used the vibrating alert from cell phones to test the degree of information reception among test subjects without increasing visual loading. Burnett and Porter (2001) showed that tactile cues can be substituted for visual cues, and can supplement auditory cues as a channel for information transmission. 
The present study uses auditory and tactile cues to replace visual cues for assistive information transmission in OBRADs to assess the impact on cyclist cognitive loading and reaction times. This study uses SDT and Likert response instrument to measure cognitive loading in detecting hazards to explore risks to cyclists as follows:

1. Assess the cognitive loading of cyclists using OBRADs;

2. Explore the potential for reducing cycling risks through the use of single-sensory /multisensory information cues.

\section{Methods Used}

This study uses signal detection theory to investigate the effects of various OBRAD information transmission modes on bicyclist behavior through lab-based simulations of authentic riding conditions.

\subsection{Experimental Setup}

To ensure consistent environmental conditions (e.g., weather or traffic), and to prevent differences in individual riding skill from affecting the results, experiments were conducted in a lab environment (see Fig. 1). The bicycle used in the experiment was a 48" steel frame road bike, which had been previously identified by Rodgers (1998) as being a type of bicycle highly prone to collisions. The bicycle was fitted to a Tacx ${ }^{\mathrm{TM}}$ FortiusMultiplayer T1930 virtual reality trainer using the Tacx 2.0 (T1990.02) software. An Epson EB410 short focus projector was positioned $230 \mathrm{~cm}$ from a $4: 3$ aspect ratio screen, which was $170 \mathrm{~cm}$ in front of the test subject's eyes. A Garmin ${ }^{\circledR}$ Edge 705 trip computer was fitted to the bicycle with text size set to $36 \mathrm{pt}, 40 \mathrm{~cm}$ from the test subject's eyes. The entire process was recorded on video to collect the following data:

1. Conditions presented on the screen

2. Average rotations per minute (RPM)

3. Head movement

4. Reaction to perceived hazard

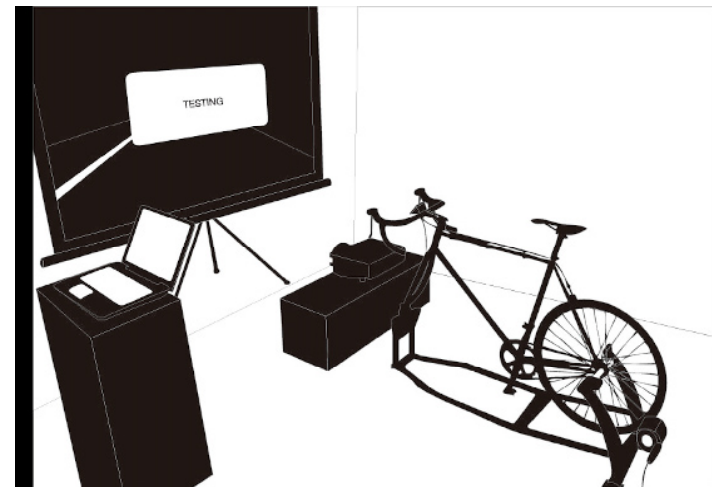

Fig. 1. Experimental setup 


\subsection{Test Subject Selection}

The experiment was conducted using 30 test subjects, including 16 male and 14 female undergraduate and graduate students from Chang Gung University, aged between 18 and 26 (mean 21.13, SD = 3.54). Test subjects ranged from 165 to $180 \mathrm{~cm}$ in height (mean 172.82, $\mathrm{SD}=4.95$ ). All test subjects had an average of 10 years or more of cycling experience, none suffered from color blindness or any other eye disease. Degrees of corrected vision ranged from 0.8 to 1.2 , and all were right handed. The test procedure lasted 90 minutes. To reduce fatigue and the memory effect, test subjects were separately tested twice. Each test subject was paid the equivalent of US\$10 after completion of the second test. Prior to testing, the bicycle seat was adjusted to suit the rider's height. To reduce psychological loading, the bicycle's gearset was fixed at $50 * 14$.

\subsection{Experimental Design}

Reaction time is the time from when the person perceives the stimulus signal to when he or she makes his or her first reaction. To calculate reaction time, we select a more clearly defined stimulus (see Table 1) (e.g., an oncoming car or a car overtaking from behind) and avoid other unnecessary stimuli. A large button is positioned on the bicycle's handlebars and test subjects can easily press the button without changing position, thus significantly reducing the physical difference in reaction behavior. Test subjects press the button in response to perceived hazard. When the button is pressed, a visible red laser dot to appear on the projection screen, and reaction time is calculated as the interval between the initial appearance of the hazard and the red dot appearing on the screen.

Table 1. Hazard stimulus definitions

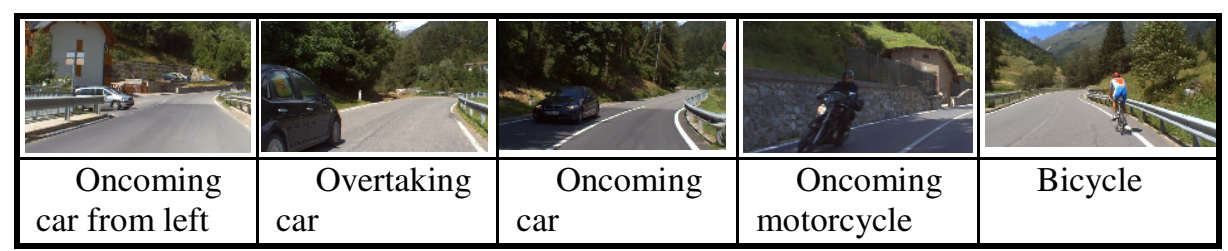

The experiment was divided into nine tasks. In each task, the test subject was asked to respond to a perceived hazard (e.g., oncoming or overtaking vehicle) by pressing the button once. Task 1 was a warm-up in which participants were asked to ride the bicycle through a rural area for three minutes. In tasks 2 through 9, riders were asked to maintain a cadence of between 55 to 65 RPM while receiving visual, auditory and tactile cues from the multimedia trip computer. Each ride simulated resistance road training with the road gradient set to within $3 \%$, and included a selection of over ten hazard stimuli distributed through each task. In the interval between tasks, test subjects were asked to provide feedback about the information they'd received from the OBRAD and road conditions in the previous task through answering short and simple 
interview questions which were recorded, and through filling out a Likert response table. These responses provided an understanding of the riders' degree of distraction while receiving information from different message modes.

\section{Experimental Results}

The operating variables used in this study include visual message reception, auditory message reception and tactile message reception.

\subsection{Cognitive Loading}

After each task the participants asked to complete a Likert response instrument to measure cognitive loading. Responses ranged from 1 (strongly disagree) to 7 (strongly agree).

Table 2. Task content of T value test

\begin{tabular}{|c|c|c|c|c|c|c|}
\hline \multicolumn{7}{|c|}{ One-Sample Test } \\
\hline & \multicolumn{6}{|c|}{ Test Value $=0$} \\
\hline & \multirow[b]{2}{*}{$t$} & \multirow[b]{2}{*}{ df } & \multirow{2}{*}{$\begin{array}{l}\text { Sig. (2- } \\
\text { tailed) }\end{array}$} & \multirow{2}{*}{$\begin{array}{c}\text { Mean } \\
\text { Difference }\end{array}$} & \multicolumn{2}{|c|}{$\begin{array}{l}\text { 95\% Confidence Interval of } \\
\text { the Difference }\end{array}$} \\
\hline & & & & & Lower & Upper \\
\hline ND & 20.696 & 29 & .000 & 4.80000 & 4.3256 & 5.2744 \\
\hline $\mathrm{v}$ & 41.713 & 29 & .000 & 6.00000 & 5.7058 & 6.2942 \\
\hline A & 11.239 & 29 & .000 & 4.43333 & 3.6266 & 5.2401 \\
\hline T & 13.681 & 29 & .000 & 3.90000 & 3.3170 & 4.4830 \\
\hline VA & 15.183 & 29 & .000 & 4.83333 & 4.1823 & 5.4844 \\
\hline VT & 14.516 & 29 & .000 & 3.96667 & 3.4078 & 4.5256 \\
\hline TA & 12.349 & 29 & .000 & 4.13333 & 3.4488 & 4.8179 \\
\hline VAT & 15.751 & 29 & .000 & 4.80000 & 4.1767 & 5.4233 \\
\hline
\end{tabular}

Table 2 shows that the various tasks have significantly different impacts $(\mathrm{P}<0.05$, $\mathrm{V}$ has the greatest impact on riding task performance $(\mathrm{M}=6.00, \mathrm{P}<0.05)$, followed by VA $(M=4.83, P<0.05)$. $\mathrm{T}$ has the smallest impact $(\mathrm{M}=3.90, \mathrm{P}<0.05)$, followed by $\mathrm{VT}$ $(\mathrm{M}=3.96, \mathrm{P}<0.05)$. This finding indicated that subjects felt the use of visual aids was more likely to affect their cycling performance and hazard perception, but that adding other information transmission forms would reduce workload. In terms of secondary aids, tactile cues produced better results than auditory inputs. Participants indicated that tactile inputs were less distracting than auditory inputs which tended to startle the rider, thus increasing mental workload and degrading primary performance.

\subsection{Hit Rate}

Fig. 2 shows that ND indicates that the test subjects maintained their RPM within the target range without using the OBRAD, while maintaining a mean hazard detection hit rate of 0.36 . Observations indicated that tactile cues had the least impact on riding safety, followed by visual cues. The results can be divided into two main groups: the first group has a higher Hit Rate, and includes Tasks ND, T, TA and VAT, while the second group has a lower Hit Rate and includes Tasks V, A, VA and VT. These results show that tactile cues can be used to effectively transmit information while 
causing a level of distraction comparable to that when no assistive devices are used (i.e., Task ND), and this lack of interference in hazard detection is worthy of further investigation. Task $\mathrm{T}$ is the only task which does not use visual cues to score a low hit rate through the use of tactile cues alone. Task VAT is the only member of the high hit rate group to use visual cues which did not provide optimal performance, which indicates that simultaneous use of all three sensory cues does not optimally affect the detection of traffic conditions, and the use of visual devices greatly reduces the rider's ability to detect hazards. Of the tests using visual cues, Task V produces a relatively high hit rate, indicating that using other sensory cues in addition to visual cues exacerbates rider distraction.

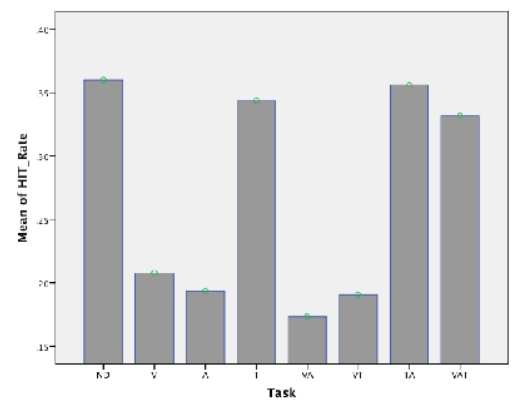

Fig. 2. Hit rate mean linear change

\subsection{Reaction Time}

Fig.3 shows that reaction time varies among the groups, with reaction times mostly averaging between $3 \mathrm{~s} \sim 4 \mathrm{~s}$. Hazard detection results are similar, and we find that Tasks $\mathrm{V}, \mathrm{A}, \mathrm{VA}$, and VT have higher reaction times $(3.47 \mathrm{~s} \sim 3.59 \mathrm{~s})$, indicating that test subjects react more slowly after becoming aware of the stimulus, while Tasks ND, T, TA and VAT have lower reaction times. Observations indicated that tactile cues had the least impact on riding safety. Using visual plus auditory cues (VA) or visual cues alone $(\mathrm{V})$, test subjects maintained a high degree of hazard perception.

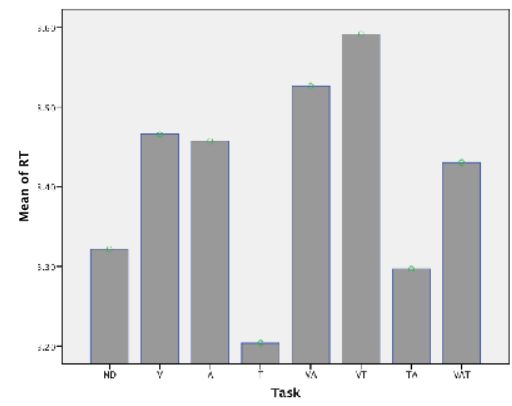

Fig. 3. Reaction time mean linear change 


\subsection{Sensitivity of d'}

Figure 4 shows the mean change of d', where Task TA has the highest sensitivity (d' mean=1.06), followed by Task $\mathrm{T}$ ( $\mathrm{d}$ ' mean=0.95), while the sensitivities of Tasks ND, A, VT and VAT are largely similar, and Tasks V and VA have relatively lower sensitivity.The d' value of Task ND is significantly lower than that of Tasks V, VA and VT. Using multiple tactile cues can lighten the rider's visual loading, thus increasing hazard detection capability. The difference between the prior hit rate and RT for Tasks $\mathrm{V}$ and VT is due to the addition of the FA variable to the value of d', which results in a reduction of the rider's mean hazard detection capability reflected in the hit rate and RT for these two tasks. However, the d' value indicates fewer misjudgments in Task VT, and more misjudgments in Task V. Task T only uses vibration cues, while Task TA uses both auditory and tactile cues, and results for both tasks indicate that the use of tactile cues effectively increases rider sensitivity to incoming information. Next, we look at Tasks $\mathrm{V}$ and VA, which exhibited a relatively low value for d'. These two tasks show that the use of visual cues corresponds to reduced rider sensitivity. Finally, Tasks ND, A, VT and VAT exhibit mid-range values for d'. Task ND uses no sensory cues, while Task A uses only auditory cues. This, plus the relatively high mean values of d' for Tasks $\mathrm{T}$ and TA, indicate that excluding visual cues results in heightened sensitivity. Results for Tasks T, VT, TA and VAT show that the use of tactile cues, regardless of whether used alone or combined with visual cues, results in a higher d' value, indicating that test subjects are more sensitive to potential risks. However, using both auditory and tactile cues for Task VA results in the lowest recorded d' values, indicating that the tactile cues are clearly more effective at conveying information than visual or auditory cues, and the inclusion of tactile cues seems to compensate for the poor performance of other types of cues.

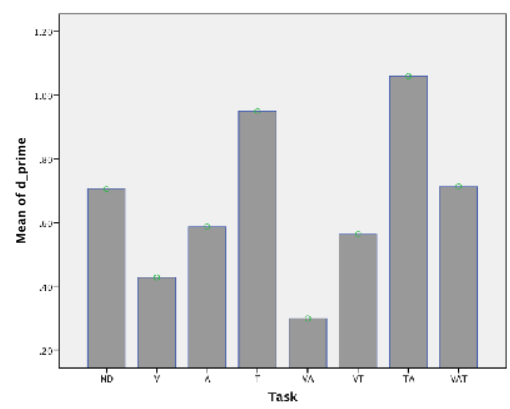

Fig. 4. d' mean linear change

\subsection{Head Movement}

Figure 5 shows that head movement in Tasks V, VA, VT and VAT can be divided into two groups, with Tasks V and VA having a higher incidence of head movement than Tasks VT and VAT. Task VA has the highest mean incidence of head movement $(m e a n=3.05)$. Given that this task involved visual and auditory cues, we can speculate 
that the use of auditory cues had a significant impact on the test subjects' reception of information and increased the number of head movements. Task VA includes auditory cues, but does not result in a higher degree of distraction than pure visual cues. However, distraction levels for Task VAT are considerably lower than for Task VT, suggesting that matching multiple sensory cues in a single application requires careful adjustment.

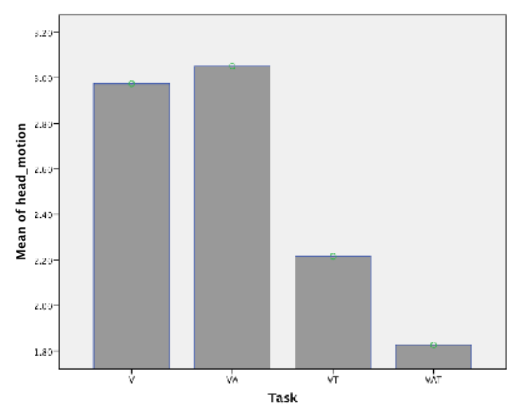

Fig. 5. Head movement mean linear change

\section{Discussion}

Cycling entails continuous posture and position adjustments which place additional stress on rider concentration capacity. Experimental results indicate that the use of visual and auditory prompts in OBRADs can lead to rider distraction and reduce rider sensitivity to potential hazards, while tactile cues are found to reduce the likelihood of distraction. The results emphasize the importance of coordinating various sensory inputs to optimize reaction time. Properly coordinating sensory cues can effectively reduce visual loading and contribute to the improved design of cycling information interfaces, thus reducing the potential for cycling accidents.

\section{Conclusion}

Safety is an important consideration for the design and use of cycling information systems. Given the high degree of environmental interference inherent in cycling, the use of existing cycling trip computers seriously exacerbates the risk of accidents. The results presented here not only contribute to the improved design of trip computer interfaces, but also provide deeper insight into rider performance, mental workload and safety. 


\section{References}

1. Bragg, B.W., Finn, P.: Influence of safety belt usage on perception of the risk of an accident. Accident Analysis \& Prevention 17(1), 15-23 (1985)

2. Brouwer, W.H., Waterink, W., Van Wolffelaar, P.C., Rothengatter, T.: Divided attention in experienced young and older drivers: lane tracking and visual analysis in a dynamic driving simulator. Human Factors: The Journal of the Human Factors and Ergonomics Society 33(5), 573-582 (1991)

3. Burnett, G.E., Mark Porter, J.: Ubiquitous computing within cars: designing controls for non-visual use. International Journal of Human-Computer Studies 55(4), 521-531 (2001)

4. Congdon, P.: VicRoads Hazard Perception Test, Can it Predict Accidents? (1999)

5. Currib, L.: The perception of danger in a simulated driving task. Ergonomics 12(6), 841849 (1969)

6. Dingus, T.A., Klauer, S.G., Neale, V.L., Petersen, A., Lee, S.E., Sudweeks, J.D., Perez, M.A., Hankey, J., Ramsey, D.J., Gupta, S., Bucher, C., Doerzaph, Z.R., Jermeland, J., Knipling, R.R.: The 100-Car Naturalistic Driving Study, Phase II - Results of the 100-Car Field Experiment (2006)

7. Doshi, A., Trivedi, M.: A comparative exploration of eye gaze and head motion cues for lane change intent prediction. 2008 IEEE Paper Presented at the Intelligent Vehicles Symposium (2008)

8. Droll, J.A.: The Velocity of Visual Attention in Vehicle Accidents. American Association for Justice (2011)

9. Eilert-Petersson, E., Schelp, L.: An epidemiological study of bicycle-related injuries. Accident Analysis \& Prevention 29(3), 363-372 (1997)

10. Gopher, G.: Attentional allocation in dual task environments. Attention and Performance III (1990)

11. Guerin, B.: What Do People Think About the Risks of Driving? Implications for Traffic Safety Interventions1. Journal of Applied Social Psychology 24(11), 994-1021 (1994)

12. Hancock, P., Verwey, W.B.: Fatigue, workload and adaptive driver systems. Accident Analysis \& Prevention 29(4), 495-506 (1997)

13. Jones, S., Furner, S.: The construction of audio icons and information cues for humancomputer dialogues. Paper Presented at the Contemporary Ergonomics: Proceedings of the Ergonomics Society's 1989 Annual Conference (1989)

14. Konstantopoulos, P., Chapman, P., Crundall, D.: Driver's visual attention as a function of driving experience and visibility. Using a driving simulator to explore drivers' eye movements in day, night and rain driving. Accident Analysis \& Prevention 42(3), 827-834 (2010)

15. Korteling, J.H.: Effects of aging, skill modification, and demand alternation on multipletask performance. Human Factors: The Journal of the Human Factors and Ergonomics Society 36(1), 27-43 (1994)

16. Lansdown, T.C., Brook-Carter, N., Kersloot, T.: Distraction from multiple in-vehicle secondary tasks: vehicle performance and mental workload implications. Ergonomics 47(1), 91-104 (2004)

17. Lee, Y.-C., Lee, J.D., Boyle, L.N.: Visual attention in driving: the effects of cognitive load and visual disruption. Human Factors: The Journal of the Human Factors and Ergonomics Society 49(4), 721-733 (2007)

18. Magill, R.A., Anderson, D.: Motor learning and control: Concepts and applications, vol. II. McGraw-Hill, New York (2007) 
19. Matanzo, F., Rockwell, T.H.: Driving performance under nighttime conditions of visual degradation. Human Factors: The Journal of the Human Factors and Ergonomics Society $9(5), 427-432(1967)$

20. Plainis, S., Murray, I.: Reaction times as an index of visual conspicuity when driving at night. Ophthalmic and Physiological Optics 22(5), 409-415 (2002)

21. Qian, H., Kuber, R., Sears, A.: Towards developing perceivable tactile feedback for mobile devices. International Journal of Human-Computer Studies 69(11), 705-719 (2011)

22. Recarte, M.A., Nunes, L.M.: Mental workload while driving: effects on visual search, discrimination, and decision making. Journal of Experimental Psychology: Applied 9(2), 119 (2003)

23. Renge, K.: Drivers'Hazard and Risk Perception, Confidence in Safe Driving, and Choice of Speed. IATSS Research 22(2), 103-110 (1998)

24. Rockwell, T.: Spare visual capacity in driving-revisited: New empirical results for an old idea. Paper Presented at the Vision in Vehicles II. Second International Conference on Vision in Vehicles (1988)

25. Rodgers, G.B.: Factors associated with the crash risk of adult bicyclists. Journal of Safety Research 28(4), 233-241 (1998)

26. Sagberg, F., Bjørnskau, T.: Hazard perception and driving experience among novice drivers. Accident Analysis \& Prevention 38(2), 407-414 (2006)

27. Salvucci, D.D.: Modeling driver distraction from cognitive tasks. Paper Presented at the Proceedings of the 24th Annual Conference of the Cognitive Science Society (2002)

28. Stanton, N.A., Salmon, P.M.: Human error taxonomies applied to driving: A generic driver error taxonomy and its implications for intelligent transport systems. Safety Science 47(2), 227-237 (2009)

29. Summala, H., Lamble, D., Laakso, M.: Driving experience and perception of the lead car's braking when looking at in-car targets. Accident Analysis \& Prevention 30(4), 401-407 (1998)

30. Tränkle, U., Gelau, C., Metker, T.: Risk perception and age-specific accidents of young drivers. Accident Analysis \& Prevention 22(2), 119-125 (1990)

31. Van Erp, J.B., Van Veen, H.A.: Vibrotactile in-vehicle navigation system. Transportation Research Part F: Traffic Psychology and Behaviour 7(4), 247-256 (2004)

32. Welford, A.T., Brebner, J.M.T.: Introduction: an historical background sketch, New York (1980) 